\title{
Valoración del residuo de piedra para preparar concreto para senderos peatonales: caso piedra Barichara
}

\author{
The Stone Waste Worthiness for the Concrete Mixtures in the \\ Pedestrian Paths: The Barichara Stone's Case
}

Valoração do resíduo de pedra para preparar concreto para
trilhas para pedestres: caso pedra Barichara

Fecha de Recepción: 10 de Mayo de 2014

María Fernanda Serrano-Guzmán*

Fecha de Aceptación: 17 de Junio de 2014

Diego Darío Pérez-Ruíz**

Mateo Sarmiento-Gómez ${ }^{* * *}$

\section{Resumen}

Los indicadores de desarrollo de un país están asociados a la satisfacción de las necesidades básicas de la población y de otras complementarias, como las relacionadas con la infraestructura vial, que favorecen la competitividad de las regiones. En algunos países en vía de desarrollo es marcado el bajo porcentaje de vías pavimentadas, con la consecuente repercusión económica que la falta de conectividad genera. Por tal razón, se hace necesario ejecutar proyectos que mejoren el estado de las vías, con alternativas que protejan la capa de afirmado.

Las lajas de piedra acomodadas con los criterios constructivos de pavimento articulado son opciones viables en aquellos sectores en donde abunda la roca. Barichara, municipio de Santander (Colombia), es una zona caracterizada por la calidad de la piedra que se extrae de manera manual y que se aprovecha para decoración y como material de revestimiento. Durante el proceso de extracción de la piedra, además de afectar la salud del personal encargado de esta labor, se genera un subproducto que impacta negativamente el paisaje. En este estudio se demuestra que la trituración del residuo del proceso de extracción de la piedra permite generar un material aprovechable en mezclas de concreto para senderos peatonales y como mortero de pega de baldosas en área de bajo tránsito.

Palabras clave: Concreto, Desarrollo Sostenible, Vías.

* Ph. D. Universidad Pontificia Bolivariana (Bucaramanga-Santander, Colombia). mariaf.serrano@upb.edu.co

** Ph. D. Pontificia Universidad Javeriana Cali (Cali-Valle del Cauca, Colombia). diegoperezr@gmail.com

*** Universidad Pontificia Bolivariana (Bucaramanga-Santander, Colombia). mateo.sarmiento@upb.edu.co 


\section{Abstract}

A country development indicators, are associated with the population's basic needs' satisfaction and other complementary needs, such as those related with the roads infrastructure, that promotes the regions' competitiveness. In some developing countries, it is marked the paved roads' low percentage, with the consequent economic impact, affected by the connectivity`s low level. For this reason, it is necessary to implement some projects which can improve the roads' conditions, with alternatives that protect the sub-base layer stated.

The stone's slabs that can be installed as an articulated pavement, becomes a viable alternative, in those territories where the stone is abundant. The Barichara, municipality of Santander (Colombia), is an area characterized by its stone's quality, which is extracted manually, and used for decoration and as a coating material. The stone's extraction process has two problems: On the one side, it affects the people involved in this work's health, on the other hand, it generates a byproduct with a negative impact in the landscape. This study shows that the crushing residue obtained after the stone extraction process, allows a usable item (residue's) generation for the concrete mixtures, both for the footpaths, and as a mortar for the tiles in a low road traffic area.

Key words: Concrete, Sustainable Development, Roads.

\section{Resumo}

Os indicadores de desenvolvimento de um país estão associados à satisfação das necessidades básicas da população e de outras complementárias, como as relacionadas com a infraestrutura rodoviária, que favorecem a competitividade das regiões. Em alguns países em via de desenvolvimento é marcada a baixa porcentagem de vias pavimentadas, com a consequente repercussão econômica que a falta de conectividade gera. Por tal razão, se faz necessário executar projetos que melhorem o estado das vias, com alternativas que protejam a camada de base.

As lajes de pedra acomodadas com os critérios construtivos de pavimento articulado são opções viáveis naqueles setores em onde abunda a rocha. Barichara, município de Santander (Colômbia), é uma zona caracterizada pela qualidade da pedra que se extrai de maneira manual e que se aproveita para decoração e como material de revestimento. Durante o processo de extração da pedra, ademais de afetar a saúde do pessoal encarregado deste trabalho, se gera um subproduto que impacta negativamente a paisagem. Neste estudo se demonstra que a trituração do resíduo do processo de extração da pedra permite gerar um material aproveitável em misturas de concreto para trilhas para pedestres e como argamassa para colar ladrilhos em área de baixo tráfego.

Palavras chave: Concreto, Desenvolvimento Sustentável, Vias. 


\section{INTRODUCCIÓN}

El acelerado desarrollo de las distintas actividades humanas está generando un incremento de la producción de residuos sólidos, cuya inadecuada disposición ocasiona grandes problemas al ambiente por la contaminación de los cuerpos de agua, el aire y el suelo, lo cual repercute en la salud de los seres vivos [1]; la falta de educación y de responsabilidad y la carencia de programas de sensibilización ambiental para separar en la fuente y recuperar materia prima para la fabricación de nuevos productos [1] son, entre otros aspectos, razones de esta problemática. Estos residuos podrían agruparse como residuos sólidos urbanos, residuos de manejo especial y residuos peligrosos [2]. Los residuos de manejo especial son los generados en los procesos productivos que no reúnen las características para ser considerados peligrosos ni como residuos sólidos urbanos o son producidos por grandes generadores [2]; los residuos de la explotación de roca y sus derivados hacen parte de este grupo y son materiales que viabilizan su uso para la construcción como material de afirmado en las vías o para concreto de andenes y sardineles [3].

El objetivo central de este artículo, además de señalar los impactos ambientales generados por la explotación manual de la piedra para revestimiento, es plantear alternativas de aprovechamiento de los residuos obtenidos por esta labor artesanal mediante una estrategia sostenible para los microempresarios del sector. Para abordar la temática se presentará el caso de uso de residuos de la explotación de la Piedra Barichara para la preparación de morteros y concretos, aplicables en senderos peatonales.

\section{ESTADO ACTUAL DE LA infraestruCtura VIAL en Colombia}

El panorama de la infraestructura de caminos en América Latina reportaba, para el año 2000, que ningún país estaba bien calificado [4], situación que ha mejorado en los últimos años según los registros del Banco Mundial [5]. Esto puede atribuirse a que durante la década de los noventa el sector de infraestructura de toda la región de América Latina y el Caribe experimentó un profundo proceso de reforma que permitió la inversión de capital privado, y para el caso concreto de Colombia, se dió inicio a las vías concesionadas.

Colombia, con 47.7 millones de habitantes, es un país que por los indicadores del 2012 fue clasificado por el Banco Mundial como de ingreso mediano-alto, con un PIB de US\$396,6 mil millones [5]. Uno de los indicadores que se considera en este informe es la infraestructura vial, que para el caso de Colombia reportaba que por lo menos en el $65 \%$ de la red vial del país (lo que representa $10.998 \mathrm{~km}$ de todas las vías) no se cumple con las condiciones de operación adecuadas para que los usuarios hagan uso de las vías con velocidades mayores a $40 \mathrm{~km} /$ hora [6].

A pesar de que la red vial primaria y algunos tramos de la red terciaria del país recibieron la construcción de vías, túneles y puentes $\mathrm{y}$ el mantenimiento $\mathrm{y}$ rehabilitación de algunos tramos [7], las evidencias señalan que las dotaciones en infraestructura del transporte son un $26 \%$ más bajas de lo que debería tener el país, posicionándolo como atrasado frente a otros países en desarrollo, como los de Asia del Este. Adicionalmente, existen situaciones, como fenómenos climáticos y altas tasas de depreciación del capital invertido, que han desestimulado las inversiones privadas, que en la actualidad representan el $40 \%$ de las inversiones totales. Así mismo, se observa que se requieren inversiones anuales del orden de 20 billones de pesos para disminuir la brecha en infraestructura vial no urbana [8].

Se debe tener en cuenta que en la medida en que las condiciones de infraestructura en bienes y servicios mejoren, mejorarán los indicadores $\mathrm{y}$, por ende, la calidad de vida de los individuos; este hecho aplica para todos los países del mundo, y con ello se garantiza el éxito de las distintas actividades manufactureras, agrícolas e industriales que se realizan en las comunidades [5]. Como consecuencia de lo anterior, surge la necesidad de plantear proyectos de mejoramiento vial acordes con la capacidad económica del país y con el uso que tengan estos tramos a nivel nacional. Alternativas como asfaltos modificados, huellas en vías secundarias y terciarias $\mathrm{y}$ adoquines, son algunas de las opciones disponibles en los municipios de Colombia. Particularmente, en el departamento de Santander (Colombia) se encuentra Barichara, municipio que provee un adoquín en piedra natural, con gran valor arquitectónico y urbanístico, 
que es apetecido para las labores de pavimentación vial en determinados sectores rurales y urbanos.

\section{A. Importancia económica de la explotación de piedra en Barichara}

Barichara es un municipio que se ha convertido en un ícono turístico en la provincia Guanentina, de Santander; teniendo en cuenta sus preciadas construcciones de finales del siglo XVIII, en 1978 el sector antiguo fue declarado patrimonio cultura. La economía del municipio se fundamenta en los renglones agrícola y ganadero, y cuenta con 354 microempresarios que desarrollan actividades secundarias, el $9.6 \%$ de ellos dedicados a la explotación de la piedra [9]. Particularmente, la piedra de la zona atrae la atención de los turistas por la calidad en el manejo de los productos que de ella se obtienen, además de las lajas para vías [10].

\section{B. Impactos ambientales de la explotación de piedra}

La explotación artesanal de la piedra (Figura 1) en la región de Barichara está generando pérdida acelerada de la cobertura vegetal, lo que afecta al paisaje, que se torna de naturaleza semidesértica. Una inspección en la zona le muestra al visitante una buena parte de material fracturado de tamaño menor a 4", que no es apto para pavimentación y que queda dispuesto en montículos en el área de trabajo (Figura 2). Adicionalmente, en la región no se lleva a cabo un programa de seguridad industrial y salud ocupacional para los habitantes que se dedican a esta labor, con los consecuentes inconvenientes que se generarán en la población que se beneficia económicamente de esta tarea. Podría agregarse también que los talladores tienen esta actividad como su estrategia de subsistencia diaria, sin un programa a futuro [3].

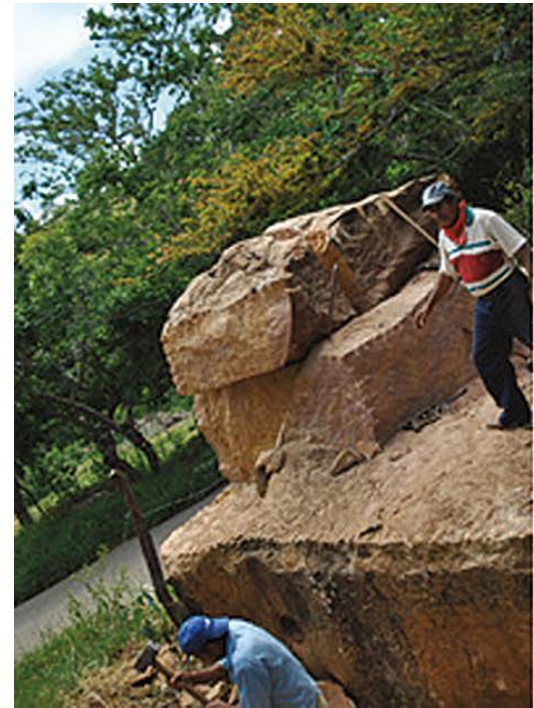

Fig. 1. Explotacion manual

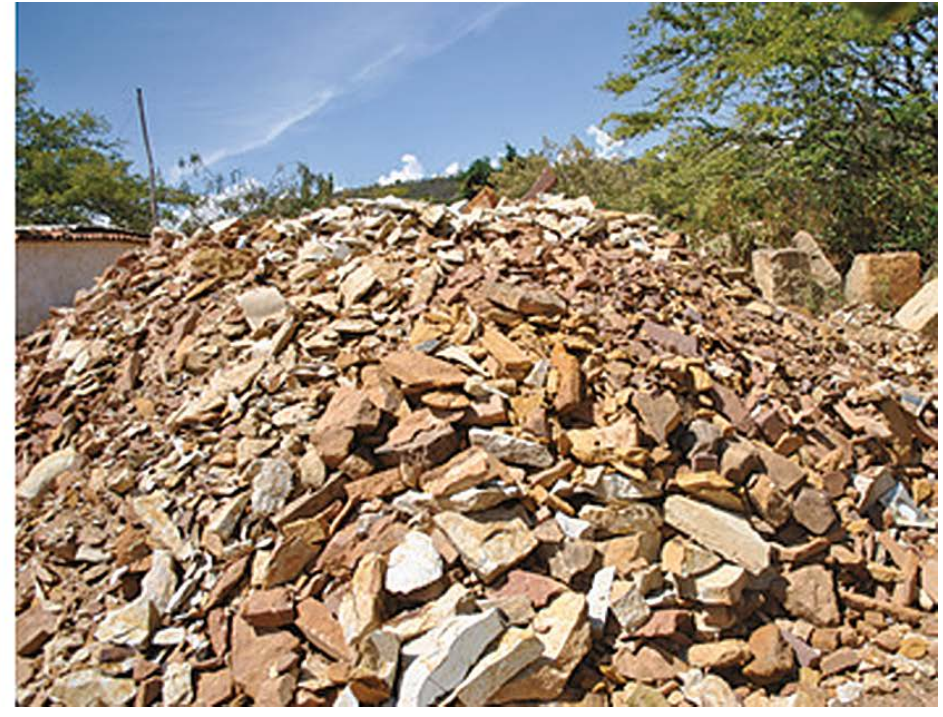

Fig. 2. Disposición desordenada del material sobrante

\section{Alternativa de aprovechamiento de la piedra Barichara}

Como se mencionó, el principal residuo luego de la extracción de las lajas corresponde a bloques de dimensiones variadas no aprovechables para los diferentes usos. Surge entonces la iniciativa del aprovechamiento de este residuo de piedra para producir materiales empleados en la preparación de morteros y concretos. Se acordó, entonces, realizar la evaluación de las propiedades mecánicas del material sobrante de la explotación de la roca para uso en morteros y concretos. 


\section{MetOdología PARA PREPARAR MORTERO Y CONCRETO CON PIEDRA EN BARICHARA}

\section{A. Selección y ajuste del tamaño del material Piedra Barichara}

En la región de Barichara existen varias canteras; para el estudio se seleccionaron las tres que reportan el mayor volumen de extracción de este material. El material recuperado, identificado como piedra
Taller, piedra Roja y piedra Blanca, fue triturado mecánicamente hasta llevarlo a un tamaño de 1".

\section{B. Caracterización del material para el estudio}

El material granular fue sometido a las pruebas de granulometría, desgaste en la máquina de los ángeles, gravedad específica y absorción de los agregados. Las normas aplicadas al material correspondieron, inicialmente, a aquellas relacionadas con la verificación del comportamiento mecánico de material granular a ser utilizado en la preparación de concretos (Figura 3).

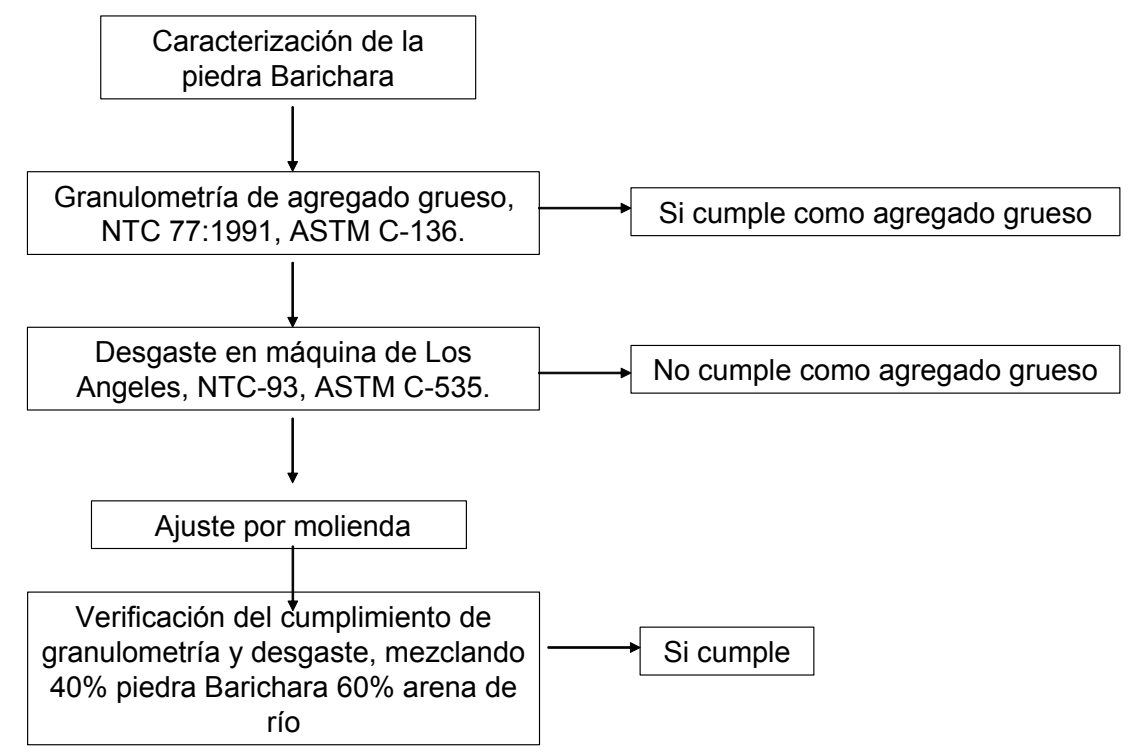

Fig. 3. Proceso de clasificación del agregado Piedra Barichara

C. Verificación del comportamiento mecánico de morteros y concretos preparados con piedra Barichara

Se planteó la elaboración de morteros utilizables en frisos y en concretos de resistencia $18 \mathrm{Mpa}$ para uso posible como material de pega y senderos peatonales. En cuanto al mortero, se siguió la norma ASTM
C109/109M, que recomienda la preparación de morteros con dosificación de cemento y arena 1:1 y una relación agua-cemento de 0.45 . Estos morteros se desencofraron de sus moldes y se colocaron en una pileta para curado por inmersión. Se prepararon cuatro tipos de mezclas con 24 especímenes de cada una que permitieron verificar la resistencia mecánica a las 24 horas, 3 días, 7 días y 28 días (Figura 4). 

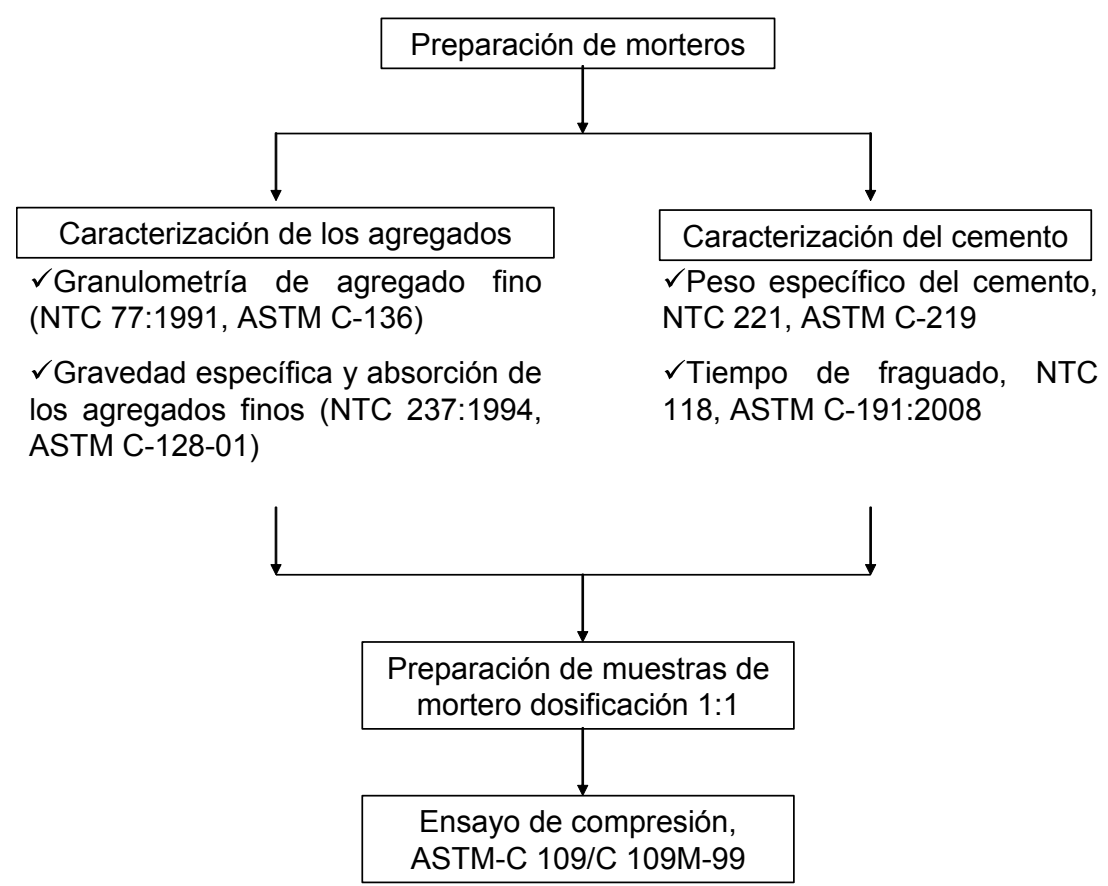

Fig. 4. Proceso para preparación de morteros

Teniendo en cuenta que la piedra Barichara reportó un desgaste superior al valor permisible de $35 \%$ para agregados para mezclas de concreto, se acordó utilizar el material granular Barichara como agregado fino, mezclándolo en proporción $40 \%$ material ajustado de Piedra Barichara y $60 \%$ arena proveniente de ríos. Se procede a realizar la mezcla de concreto según los métodos de la American Concrete Institute, preparando 72 especímenes que fueron ensayados a los 7, 14 y 28 días (Figura 5). 


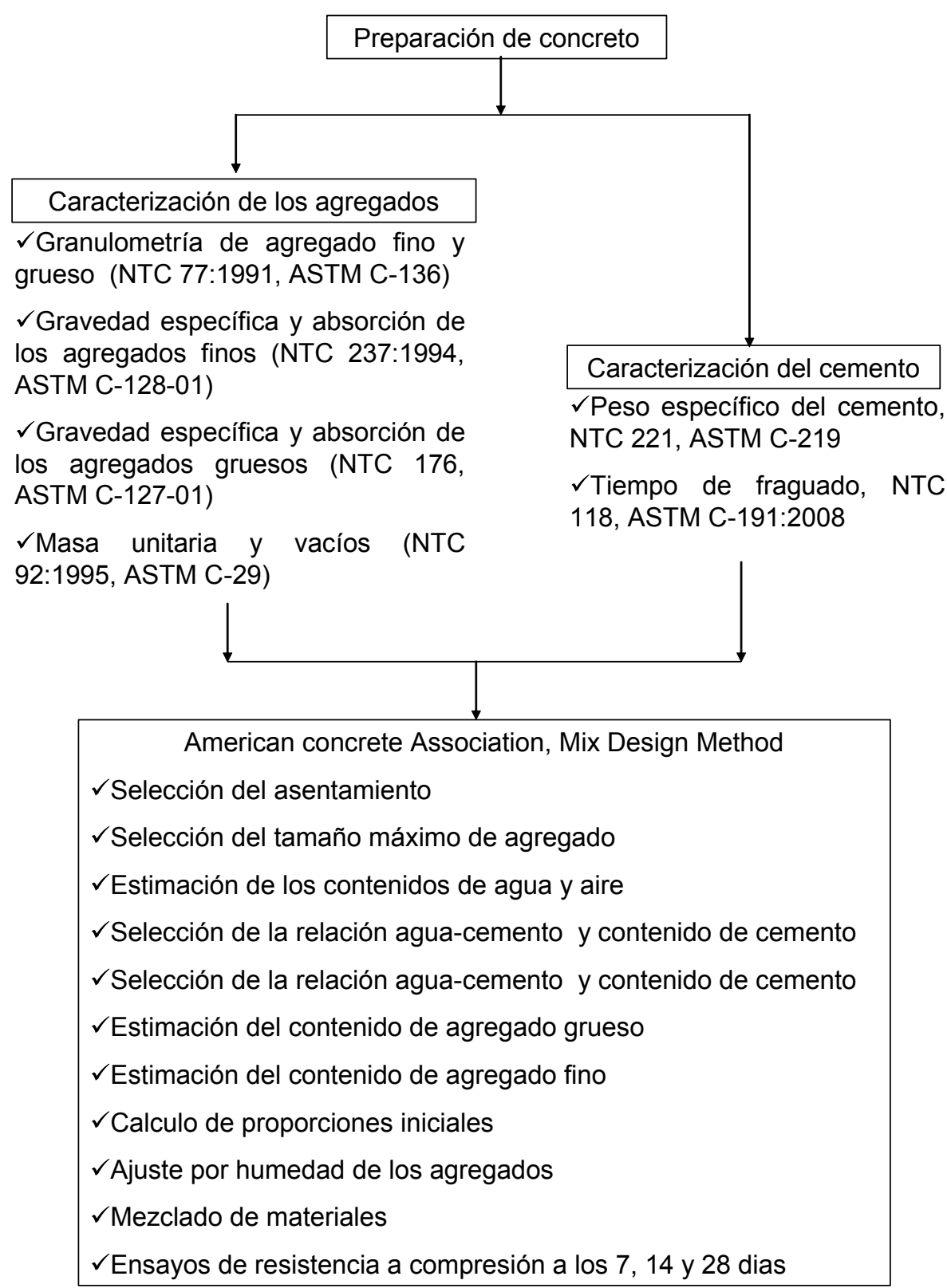

Fig. 5. Dosificación de mezclas de concreto

\section{Resultados}

La pretensión inicial de emplear los residuos de la Piedra Barichara como material agregado grueso fue descartada por cuanto los porcentajes de desgaste encontrados en el material Taller, Roja y Blanca superaron el 35\%, que es el límite de desgaste permitido para agregados a ser utilizados en concretos (NTC 93:1995, ASTM C-535). Por esta razón, se optó por trabajar con el material con la clasificación como agregado fino. Para el ajuste de tamaños se hizo pasar el material por la máquina de Los Ángeles, simulando un proceso de trituración mecánica aplicable en una cantera.

\section{A. Con respecto al material granular}

El material granular extraído de Barichara fue sometido a pruebas convencionales para verificación de calidad en morteros y concretos; esto implicó el 
ajuste de tamaños mediante trituración, de lo cual se logró identificar material grueso y material fino; sin embargo, el aprovechamiento del material grueso como triturado para concretos fue descartado por cuanto su desgaste en la máquina de Los Ángeles arrojó valores por encima de $67.20 \%$, lo cual estaba fuera de las normas de calidad. Se observó también que producto de la trituración mecánica se generaba un porcentaje significativo de material fino, al cual se le realizó la granulometría correspondiente, que se demuestra en la Figura 6.

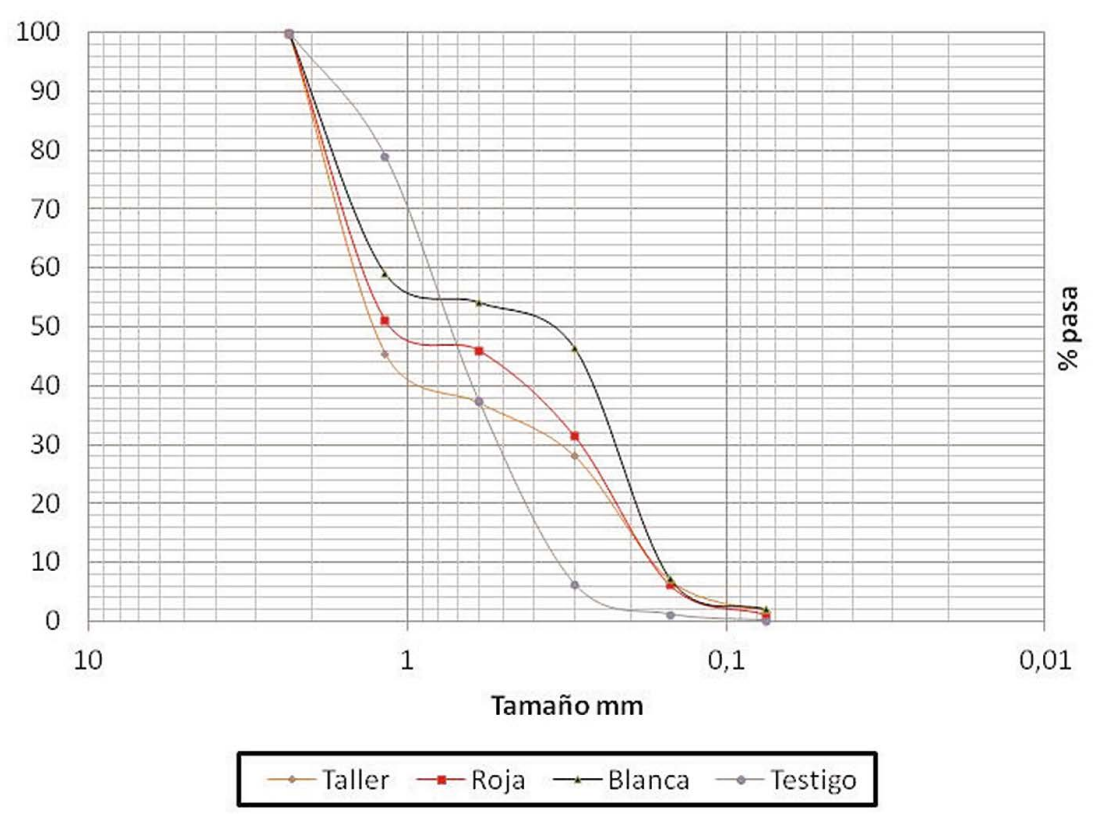

Fig. 6. Curva granulométrica del material pétreo empleado en la preparación de morteros

En este caso, el comportamiento de los materiales provenientes de Barichara presentó una distribución de tamaños por encima y por debajo de $0.6 \mathrm{~mm}$, lo que permitió reconocer una gradación que favorecía la preparación de los morteros. Se destaca también que la gravedad específica de las muestras Barichara osciló entre $2.56 \mathrm{~g} / \mathrm{cm}^{3}$ y $2.70 \mathrm{~g} / \mathrm{cm}^{3}$, mientras que las testigo arrojaron $2.71 \mathrm{~g} / \mathrm{cm}^{3}$; en todos los casos los valores de la gravedad específica correspondieron a gravedades de las arenas utilizadas para mortero y concreto en la región.

\section{B. Con respecto al cemento}

La gravedad específica del cemento fue de $2,75 \mathrm{~g} /$ $\mathrm{cm}^{3}$, que corresponde a las condiciones regulares de cementos producidos en la región, y el tiempo de fraguado inicial fue de 200 min., lo cual también es consistente con lo esperado en este tipo de cemento, indicando aceptación de su uso para morteros y concretos.

\section{Con respecto al comportamiento a compresión de los morteros preparados}

Los morteros preparados con arena proveniente de río (testigo) arrojaron, a las 24 horas y 3 días, resistencias superiores a los preparados con arenas de Barichara (Taller, Blanca y Roja); sin embargo, a los 7 días, la resistencia mecánica de los morteros preparados con arena Barichara Blanca superaron la resistencia del testigo en $8.1 \%$.

Se destaca que en todos los casos la resistencia mecánica fue superior a la resistencia de diseño (18 Mpa) a los 7 días, y que con excepción de la Roja, todos los demás especímenes reportaron resistencias mayores a los 28 días. Con estos resultados se comprueba la viabilidad del uso de la piedra Barichara triturada para morteros (Figura 7). 


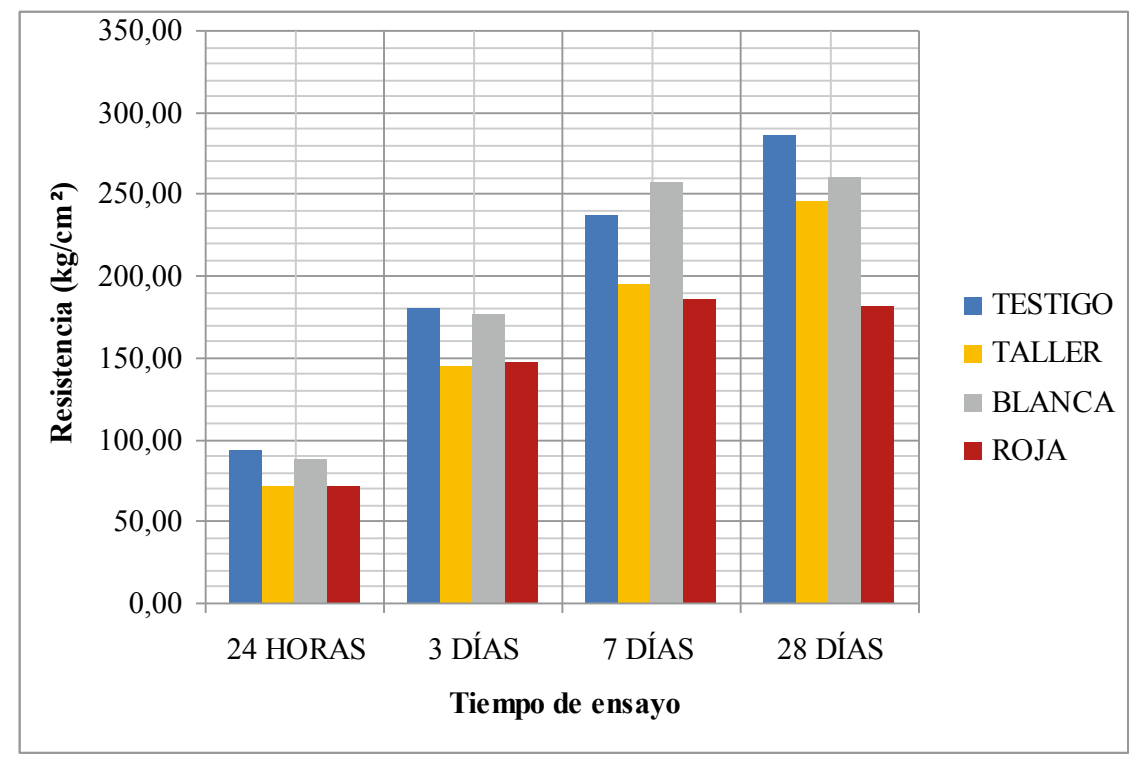

Fig. 7. Ensayos a compresión de los especímenes de mortero

En la Tabla 1 se presenta el comportamiento mecánico de las distintas propiedades evaluadas.

\section{TABLA 1}

RESULTADOS DE LAS PRUEBAS MECÁNICAS PARA ACEPTACIÓN DE AGREGADOS PARA CONCRETOS SEGÚN LOS ESTÁNDARES DE CALIDAD COLOMBIANOS

\begin{tabular}{|c|c|c|c|c|c|c|}
\hline \multirow{2}{*}{\multicolumn{2}{|c|}{ ENSAYOS REALIZADOS }} & \multicolumn{5}{|c|}{ MUESTRA } \\
\hline & & TALLER & ROJA & BLANCA & TESTIGO & REQUISITO \\
\hline \multicolumn{2}{|c|}{ Porcentaje de absorción(\%) } & 2 & 4 & 2 & 1 & - \\
\hline \multicolumn{2}{|c|}{ Gravedad específica (g/cm3) } & 2.7 & 2.56 & 2.64 & 2.71 & - \\
\hline \multicolumn{2}{|c|}{ Módulo de finura } & 2.82 & 2.6 & 2.3 & 2.76 & 2.3 a 3.1 \\
\hline \multicolumn{2}{|c|}{ Humedad natural $(\%)$} & 0.6 & 2.9 & 2.5 & 2 & - \\
\hline \multirow{2}{*}{$\begin{array}{l}\text { Masa unitaria }(\mathrm{g} / \\
\mathrm{cm} 3 \text { ) }\end{array}$} & Suelta & 1.63 & 1.57 & 1.55 & 1.48 & - \\
\hline & Compacta & 1.74 & 1.72 & 1.75 & 1.57 & - \\
\hline \multicolumn{2}{|c|}{\begin{tabular}{|l|l} 
aumento de masa unitaria (\%) \\
\end{tabular}} & 6.32 & 8.72 & 11.43 & 5.73 & - \\
\hline
\end{tabular}

El comportamiento mecánico a compresión en los morteros ensayados a los 28 días demostró resistencias por encima de los $18 \mathrm{Mpa}$ (Tabla 2). En cuanto a los concretos, la mezcla de piedra Barichara con agregado natural mejoró la resistencia de los concretos preparados con relación agua/cemento 0.47 .

\section{TABLA 2}

RESULTADO A LOS 28 DÍAS EN MORTEROS Y CONCRETOS PREPARADOS CON LA MEZCLA 60\%40\%, RELACIÓN AGUA/CEMENTO 0,47

\begin{tabular}{|l|c|c|c|c|}
\hline Muestra & \multicolumn{2}{|c|}{ Mortero (Mpa) } & \multicolumn{2}{c|}{ Concreto (Mpa). } \\
\hline & Diseño & Obtenido & Diseño & Obtenido \\
\hline Testigo & 18 & 28,7 & 18 & 17,3 \\
\hline Taller & & 24,7 & & 18,2 \\
\hline Roja & & 26,1 & & 18,3 \\
\hline Blanca & & 18,2 & & 19,7 \\
\hline
\end{tabular}




\section{Conclusiones}

Existen alternativas para mejorar la infraestructura vial, como las huellas en concreto, el riego asfáltico con mezclas bituminosas modificadas y los adoquines, entre otros. Los adoquines son elementos que ayudan a conformar un pavimento y pueden ser elaborados en plástico, concreto o piedra. En aquellas regiones en donde es viable el uso de lajas de piedra para pavimentación, la explotación manual del material genera residuos que impactan el ecosistema, por los cambios negativos en el paisaje y las afectaciones en la salud de las personas y los animales, repercusiones ocasionadas por la libre disposición del material residual obtenido.

Barichara es una región cuyo sustento económico está asociado a la explotación de la piedra para uso ornamental en viviendas o para aplicaciones estructurales en revestimiento de andenes y vías. Las labores propias de extracción del material están generando unos residuos que actualmente no se están aprovechando. En este estudio se propuso el uso del material sobrante, luego de trituración, para la preparación de morteros para frisos y concretos para andenes. De acogerse esta iniciativa, es necesario iniciar un programa de concienciación de un sistema de producción sostenible que proteja los individuos y el entorno.

\section{Agradecimientos}

A la Dirección General de Investigaciones y a Ecopetrol-ICP, en el convenio de colaboración con la Universidad Pontificia Bolivariana, Seccional Bucaramanga, 2013.

A la comunidad de Barichara y Villanueva.

Al señor Antonio Sarmiento, por el servicio de trituración, y a la señora Paulina Becerra, propietaria de las canteras.

Al escultor Miguel Ángel Viviescas, dueño del taller artesanal.

\section{REFERENCIAS}

[1] O. Castrillón Quintana and S. M. Puerta Echeverri. Impacto del manejo integral de los residuos sólidos en la Corporación Universitaria Lasallista [Impact of integral management of solid waste in the Corporación Universitaria Lasallista]. Revista de investigación Lasallista. Vol. 1, N. ${ }^{\circ}$ 1, junio, pp. 15-21, 2004.

[2] D. Ibarra Rodríguez, P. Hernández Rosales, M. T. Oropeza and C.E. Saldaña Durán. Evaluación física y química de lixiviados de residuos de manejo especial, de la empresa REDESO en Tepic, Nayarit. Memorias del Sexto Encuentro de Residuos Sólidos. ISBN. 978-607-96258-01, pp. 163-169, Nayarit, México, septiembre, 2013.

[3] M. F. Serrano Guzmán, D. D. Pérez Ruiz, M. Sarmiento Gómez and C. Toloza. Aprovechamiento de residuos de explotación de roca para preparación de materiales de construcción. Memorias del Sexto Encuentro de Residuos Sólidos. ISBN. 978-607-96258-01, pp. 230-235. Nayarit, México, septiembre, 2013.

[4] P. Millán Smitmans. Panorama del sector transportes en América Latina y el Caribe. Documento de Trabajo N. ${ }^{\circ} 1$. Pontificia Universidad Católica de Argentina. pp. 49. Noviembre, 2005.

[5] http://datos.bancomundial.org/indicador/ IS.ROD.PAVE.ZS, [consultado el 2 de marzo de 2014].

[6] Redacción Vehículos. Estado de vías en Colombia solo da para ir a $40 \mathrm{~km} / \mathrm{h}$. Nota Interior. Disponible en: http://www.eltiempo.com/ colombia/ARTÍCULO-WEB- NEW NOTA INTERIOR-13147884.html [consultado el $2 \overline{8}$ de octubre del 2013].

[7] J. M. Santos. Rendición de cuentas. Informe al Congreso de Colombia. p. 235. Bogotá. 
[8] Elespectador.com. Colombia es uno de los países más atrasados en infraestructura vial. Disponible en http://www.elespectador.com/noticias/ nacional/articulo-388585-colombia-uno-delos-paises-mas-atrasados-infraestructuravial, publicado el 22 de noviembre de 2012. [Consultado el 2 de marzo de 2014].

[9] Esquema de Ordenamiento Territorial. Barichara con su pasado ordena su futuro. Documento Gubernamental, 2013.
[10] Sistema Nacional de Información Cultural. Conjuntos Urbanos Históricos-Centro Histórico de Barichara. [Consultado el 20 de abril de 2012].

[11] C. A. Toloza. Evaluación del comportamiento físico-mecánico de la piedra de Barichara como agregado en la preparación de mezclas de concreto. BS thesis. Universidad Pontificia Bolivariana. Bucaramanga, Santander, Colombia, 2013. 